\title{
Segurança do paciente na atenção primária à saúde: uma reflexão teóríca
}

\author{
Patient safety in primary health care: a theoretical reflection \\ Seguridad del paciente em la atención primaria de salud: uma reflexión teórica
}

\begin{abstract}
RESUMO
Objetivo: Analisar, a partir de uma reflexão teórica, os principais eventos adversos e o contexto da segurança do paciente na atenção primária à saúde. Método: Trata-se de um estudo qualitativo, descritivo do tipo teórico-reflexivo desenvolvido a partir da abordagem de revisão narrativa com artigos científicos nacionais e internacionais disponíveis nas bases de dados LILACS, BDENF, SCiELO e MEDLINE. Resultados: Identificou-se que as pesquisas relacionadas à segurança do paciente estão predominantemente direcionadas para a assistência hospitalar. Contudo, os principais eventos adversos ocorridos na atenção primária estão relacionados ao atraso diagnóstico, medicação, tratamento odontológico, injeções, vacinas, comunicação entre médico e pacientes, documentação e processos administrativos. Conclusão: Tem-se a necessidade de ampliar as discussões sobre segurança do paciente na atenção primária de modo a possibilitar que pacientes e profissionais possam reconhecer e gerenciar os eventos adversos e serem sensiveis à sua capacidade compartilhada para a mudança reduzindo erros e tensões.
\end{abstract}

DESCRITORES: Segurança do paciente; Atenção primária à saúde; Qualidade da assistência à saúde.

\section{ABSTRACT}

Objective: To analyze, from a theoretical reflection, the main adverse events and the context of patient safety in primary health care. Method: This is a qualitative, descriptive study of the theoretical-reflective type developed from the narrative review approach with national and international scientific articles available in the LILACS, BDENF, SciELO and MEDLINE databases. Results: It was found that research related to patient safety is predominantly directed towards hospital care. However, the main adverse events that occurred in primary care are related to delayed diagnosis, medication, dental treatment, injections, vaccines, communication between doctor and patients, documentation and administrative processes. Conclusion: There is a need to expand discussions on patient safety in primary care in order to enable patients and professionals to recognize and manage adverse events and be sensitive to their shared capacity for change, reducing errors and tensions.

ESCRIPTORS: Patient safety; Primary health care; Quality of health care.

\section{RESUMEN}

Objetivo: Analizar, desde una reflexión teórica, los principales eventos adversos y el contexto de la seguridad del paciente en la atención primaria de salud. Método: Se trata de un estudio cualitativo, descriptivo de tipo teórico-reflexivo desarrollado a partir del enfoque de revisión narrativa con artículos científicos nacionales e internacionales disponibles en las bases de datos LILACS, BDENF, SciELO y MEDLINE. Resultados: Se encontró que la investigación relacionada con la seguridad del paciente se dirige predominantemente hacia la atención hospitalaria. Sin embargo, los principales eventos adversos que ocurrieron en atención primaria están relacionados con el retraso en el diagnóstico, medicación, tratamiento odontológico, inyecciones, vacunas, comunicación entre médico y pacientes, documentación y procesos administrativos. Conclusión: Es necesario ampliar los debates sobre la seguridad del paciente en atención primaria para que los pacientes y los profesionales puedan reconocer y gestionar los eventos adversos y ser sensibles a su capacidad compartida de cambio, reduciendo errores y tensiones.

DESCRIPTORES: Seguridad del paciente; Atención primaria de salud; Calidad de la atención de salud.

RECEBIDO EM: 27/10/2020 APROVADO EM: 04/11/2020

\section{Ricardo Saraiva Aguiar}

Universidade Católica de Brasília (UCB). Escola de Saúde e Medicina, Programa de Pós-Graduação em Gerontologia, Brasília-DF, Brasil.

ORCID: 0000-0003-0335-2194 


\section{Henrique Salmazo da Silva}

Universidade Católica de Brasília (UCB). Escola de Saúde e Medicina, Programa de Pós-Graduação em Gerontologia, Brasília-DF, Brasil.

ORCID: 0000-0002-3888-4214

\section{INTRODUÇÃO}

A qualidade do cuidado está presente na agenda de saúde global, sendo a segurança do paciente um dos seus componentes ${ }^{1}$, pois compreende ações voltadas ao gerenciamento e à prevenção de risco e exposição durante a assistência à saúde prestada a pacientes em variados contextos e necessidades. ${ }^{2}$

Para o alcance desse cuidado, dito como seguro, as instituições de saúde têm se esforçado em melhorar os processos de cuidado oferecidos aos usuários, reconhecendo em primeira instância a necessidade de se estabelecer a cultura de segurança do paciente como rotina do serviço de saúde.-3

Essa passou a estar mais presente nas pautas das agendas dos serviços de saúde públicos e privados após a publicação em 1999 do relatório norte-americano "Errar é humano: construindo um sistema de saúde mais seguro" do Instituto de Medicina (IOM) ${ }^{4-5} \mathrm{O}$ documento apresenta os resultados do estudo de prontuários que apontou a ocorrência de iatrogenias em um pouco mais de $3 \%$ do total analisado. Além disso, foram documentados de 44 a 98 mil óbitos de pacientes por ano nos Estados Unidos da América (EUA). Grande parcela deles poderia ter sido evitada com a adoção de práticas voltadas a promoção de um cuidado seguro. ${ }^{4}$

Desse modo, entende-se que devido à diversidade de situações de saúde que são solucionadas na Atenção Primária à Saúde (APS), há uma exigência dos profissionais de saúde para que tenham habilidade, conhecimento técnico e atitudes de empatia que envolvam a escuta qualificada e a personalização do atendimento. No entanto, a atuação profissional na assistência direta é passível de falhas, sejam elas técnicas, sejam provenientes da ausência de atitudes de empatia. ${ }^{6}$

A APS é definida como um conjunto de valores, de princípios e de atributos estruturantes, devendo ser a base dos sistemas de saúde por ser a melhor estratégia para produzir melhorias sustentáveis e maior equidade no estado de saúde de uma população. ${ }^{7-8}$ Foi proposta para a organização de serviços com foco nas necessidades de saúde da população adscrita e baseada em uma ação multiprofissional e interprofissional com participação social. ${ }^{9}$

Pode-se pensar que a APS, por não atuar diretamente com procedimentos de alta tecnologia, seja um ambiente protegido e seguro, porém a ocorrência de eventos adversos também está presente nesse nível de atenção, fato identificado em estudo realizado no Brasil que mostrou a incidência de $82 \%$ de danos que atingiram os pacientes, muitos deles com gravidade muito alta - dano permanente (25\%) ou óbito (7\%). Entre os erros identificados que contribuíram com os eventos adversos, destacam-se: erros no atendimento administrativo, na investigação de exames, com tratamentos, de comunicação, na gestão de recursos humanos, na execução de uma tarefa clínica e de diagnóstico. ${ }^{10-11}$

Embora os incidentes relativos à APS possam ser menos prejudiciais do que alguns dos que ocorrem em hospitais, estes podem ser de grande magnitude, devido à elevada quantidade de usuários atendidos, de serviços e de procedimentos realizados ao logo da vida das pessoas nesse nível da rede de atenção. ${ }^{12} \mathrm{~A}$ APS é considerada a coordenadora do cuidado e a porta de entrada preferencial dos usuários aos diferentes pontos da atenção da redes de atenção à saúde (RAS). ${ }^{13}$ Apesar disso, o tema da segurança do paciente na APS não foi explorado na mesma medida que nos ambientes hospitalares, sendo ainda incipiente na literatura. ${ }^{10,12}$

Dessa forma, o emprego de ações específicas visando à segurança do cuidado é recomendado de modo a padronizar os processos de trabalho, a identificação dos riscos, o planejamento da assistência, o comprometimento gerencial, a comunicação entre os profissionais e a implementação de uma cultura favorável à educação e à mudança de condutas cujo foco é a redução dos eventos adversos. ${ }^{13}$

Portanto, este artigo tem o objetivo de analisar, a partir de uma reflexão teórica, os principais eventos adversos e o contexto da segurança do paciente na atenção primária à saúde.

\section{MÉTODOS}

Trata-se de um estudo qualitativo, descritivo do tipo teórico-reflexivo desenvolvido a partir da abordagem de revisão narrativa com artigos científicos nacionais e internacionais que abordam sobre a segurança do paciente e eventos adversos no contexto da APS.

Realizou-se, para a busca das produções, a seguinte pergunta de pesquisa: "Qual a produção, na literatura, sobre a segurança do paciente e eventos adversos no contexto da APS?". Selecionavam-se, assim, os artigos que abordavam a segurança do paciente e eventos adversos no contexto da APS.

Os artigos foram pesquisados no período de janeiro a março de 2020 por meio das bases de dados Literatura Latino-Americana e do Caribe em Ciências da Saúde (LILACS), Medical Literature Analysis and Retrieval System Online (MEDLINE), Base de Dados em Enfermagem (BDENF) e Scientific Eletronic Library Online (SciELO), refinando a busca para o período de 2008 a 2020 e utilizando os seguintes critérios de inclusão: artigos disponíveis de forma on line; nos idiomas português, inglês ou espanhol; e pesquisas originais ou de revisão de literatura.

Dividiu-se o estudo em etapas: pesquisa dos artigos nas bases de dados; leitura dos títulos e dos resumos para verificar a convergência do material à temática de estudo e aos critérios de inclusão; leitura 
do artigo na íntegra; busca e leitura dos estudos originais encontrados por meio das referências finais dos artigos oriundos da busca nas bases de dados. Realizaram-se, após todas as leituras, a compilação dos materiais, seguida da análise e identificação dos eventos adversos nos idosos para a reflexão e, finalmente, a elaboração das sínteses reflexivas do estudo.
Com os artigos selecionados, utilizou-se a síntese narrativa para análise e discussão dos dados.

\section{RESULTADOS}

Foram utilizados 12 nesta revisão narrativa (Quadro 1), sendo interpretados e sintetizados os resultados através de uma comparação dos dados evidenciados na análise dos artigos.

\section{DISCUSSÃO}

A prestação de cuidados em saúde é bastante complexa e pressupõe o necessário equilíbrio entre benefícios e danos que acompanham todo processo, a fim de pro-

Quadro 1 - Distribuição dos artigos de acordo com o periódico, ano de publicação, autor(es), título, delineamento e resultados. Brasilia, Distrito Federal, Brasil, 2020.

\begin{tabular}{|c|c|c|c|c|c|}
\hline & $\begin{array}{l}\text { PERIÓDICO E ANO DE } \\
\text { PUBLICAÇÃO }\end{array}$ & AUTOR(ES) & TÍTULO & DELINEAMENTO & RESULTADOS \\
\hline 亯 & $\begin{array}{l}\text { Revista Gaúcha de En- } \\
\text { fermagem, } 2019\end{array}$ & $\begin{array}{l}\text { Silva APF, Backes } \\
\text { DS, Magnago TSBS, } \\
\text { Colomé JS }\end{array}$ & $\begin{array}{l}\text { Segurança do pacien- } \\
\text { te na atenção primária: } \\
\text { concepções de enfer- } \\
\text { meiras da Estratégia } \\
\text { Saúde da Famillia }\end{array}$ & Qualitativo & $\begin{array}{l}\text { As enfermeiras relacionam a se- } \\
\text { gurança do paciente a atitudes que } \\
\text { não provoquem maiores danos ao } \\
\text { usuário. Dentre as dificuldades en- } \\
\text { contradas, destacam-se: estrutura } \\
\text { física inadequada, falta ou deficiên- } \\
\text { cia de material de consumo, falhas } \\
\text { relativas à gestão e ou organização } \\
\text { e sobrecarga de trabalho. }\end{array}$ \\
\hline $\begin{array}{l}N \\
\text { O } \\
\text { 量 } \\
\text { 妾 }\end{array}$ & $\begin{array}{l}\text { Journal of Nursing and } \\
\text { Health, } 2018\end{array}$ & $\begin{array}{l}\text { Souza LM, Silva } \\
\text { MCS, Zavalhia SR, } \\
\text { Coppola IS, Rocha } \\
\text { BP }\end{array}$ & $\begin{array}{l}\text { Percepção de enfermei- } \\
\text { ros da Estratégia Saúde } \\
\text { da Familia sobre segu- } \\
\text { rança do paciente }\end{array}$ & Qualitativo & $\begin{array}{l}\text { Identificou-se falta de familiariza- } \\
\text { ção dos enfermeiros com o assunto. } \\
\text { Erros de medicação e quedas foram } \\
\text { problemas à segurança do paciente } \\
\text { e a comunicaçãa efetiva como fator } \\
\text { promotor. A capacitação das equi- } \\
\text { pes, implementação de instrumen- } \\
\text { tos próprios voltados à segurança } \\
\text { do paciente e diminuição de traba- } \\
\text { Iho foram apontadas enquanto es- } \\
\text { tratégias de melhoria. }\end{array}$ \\
\hline 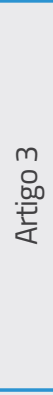 & $\begin{array}{l}\text { Research, Society and } \\
\text { Development, } 2020\end{array}$ & Alves AS, Aguiar RS & $\begin{array}{l}\text { Segurança do paciente } \\
\text { no âmbito domiciliar: } \\
\text { uma revisão integrativa }\end{array}$ & $\begin{array}{l}\text { Revisão integra- } \\
\text { tiva }\end{array}$ & $\begin{array}{l}\text { A segurança no âmbito domiciliar } \\
\text { constitui importante objeto de es- } \\
\text { tudo, devendo ter sua relevância } \\
\text { adequadamente compreendida } \\
\text { por profissionais de enfermagem, } \\
\text { cuidadores e familiares, visando o } \\
\text { desenvolvimento de práticas ade- } \\
\text { quadas de cuidado que possam } \\
\text { proporcionar a construção de um } \\
\text { ambiente seguro. }\end{array}$ \\
\hline 党 & $\begin{array}{l}\text { Caderno de Saúde Pú- } \\
\text { blica, } 2014\end{array}$ & $\begin{array}{l}\text { Marchon SG, Men- } \\
\text { des Júnior WV }\end{array}$ & $\begin{array}{l}\text { Segurança do paciente } \\
\text { na atenção primária à } \\
\text { saúde: revisão sistemá- } \\
\text { tica }\end{array}$ & $\begin{array}{l}\text { Revisão sistemá- } \\
\text { tica }\end{array}$ & $\begin{array}{l}\text { O método mais utilizado nos estu- } \\
\text { dos foi a análise de incidentes em } \\
\text { sistemas de notificação de inciden- } \\
\text { tes, sendo que os tipos de inciden- } \\
\text { tes mais encontrados na atenção } \\
\text { primária estavam associados à } \\
\text { medicação e diagnóstico. Os fatores } \\
\text { contribuintes mais relevantes fo- } \\
\text { ram falhas de comunicação entre os } \\
\text { membros da equipe de saúde. }\end{array}$ \\
\hline
\end{tabular}




\section{artigo}

Aguiar, R.S.; Salmaza da Silva, $H$

Segurança do paciente na atenção primária à saúde: uma reflexão teórica

\begin{tabular}{|c|c|c|c|c|c|}
\hline 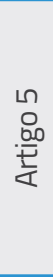 & $\begin{array}{l}\text { Cadernos de Saúde Pú- } \\
\text { blica, } 2015\end{array}$ & $\begin{array}{l}\text { Marchon SG, Men- } \\
\text { des Júnior WV, Pa- } \\
\text { vão ALB }\end{array}$ & $\begin{array}{l}\text { Características dos } \\
\text { eventos adversos na } \\
\text { atenção primária à saúde } \\
\text { no Brasil }\end{array}$ & Prospectivo & $\begin{array}{l}\text { A taxa de incidentes que não atingiram } \\
\text { os pacientes foi de } 0,11 \% \text {. A taxa de } \\
\text { incidência de incidentes que atingiram } \\
\text { os pacientes, mas não causaram dano } \\
\text { foi de } 0,09 \% \text {. A taxa de incidência de in- } \\
\text { cidentes que atingiram os pacientes e } \\
\text { causaram evento adverso foi de } 0,9 \% \text {. }\end{array}$ \\
\hline 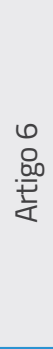 & $\begin{array}{l}\text { BMJ Quality \& Safety, } \\
2011\end{array}$ & Wallis K, Dovey S & $\begin{array}{l}\text { No-fault compensation } \\
\text { for treatment injury in } \\
\text { New Zealand: identifying } \\
\text { threats to patient safety } \\
\text { in primary care }\end{array}$ & Quantitativo & $\begin{array}{l}\text { Houve } 6007 \text { reclamações de lesões } \\
\text { por tratamento de cuidados primários; } \\
64 \% \text { foram aceitos como lesões de tra- } \\
\text { tamento. A maioria das reclamações } \\
\text { foi avaliada como menor }(83 \%), 12 \% \\
\text { maior, } 4 \% \text { grave e } 1 \% \text { sentinela. Os me- } \\
\text { dicamentos causaram a maioria das } \\
\text { lesões }(38 \%) \text { e as lesões mais graves e } \\
\text { sentinela }(60 \%) \text {. }\end{array}$ \\
\hline 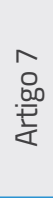 & $\begin{array}{l}\text { Quality Safety Health } \\
\text { Care, } 2010\end{array}$ & $\begin{array}{l}\text { Mira JJ, Nebot C, Lo- } \\
\text { renzo S, Pérez-Jover } \\
\text { V }\end{array}$ & $\begin{array}{l}\text { Patient report on infor- } \\
\text { mation given, consulta- } \\
\text { tion time and safety in } \\
\text { primary care }\end{array}$ & Quantitativo & $\begin{array}{l}\text { O tempo de consulta, a rotatividade do } \\
\text { medico e informações sobre as pre- } \\
\text { cauções de tratamento determinam } \\
\text { o maior risco de reações adversas ao } \\
\text { tratamento. }\end{array}$ \\
\hline $\begin{array}{l}\infty \\
0 \\
0 \\
.0 \\
\text { 尊 }\end{array}$ & $\begin{array}{l}\text { BMJ Quality \& Safety, } \\
2011\end{array}$ & $\begin{array}{l}\text { O'Beirne } \mathrm{M} \text {, Sterling } \\
\text { PD, Zwicker K, He- } \\
\text { bert P, Norton PG }\end{array}$ & $\begin{array}{l}\text { Safety incidents in family } \\
\text { medicine }\end{array}$ & Qualitativo & $\begin{array}{l}\text { A grande maioria dos incidents foi } \\
\text { considerado como evitáveis. Danos fo- } \\
\text { ram associados a } 50 \% \text { dos incidentes. } \\
\text { Apenas } 1 \% \text { dos incidentes tiveram um } \\
\text { impacto severo. Os quatro principais } \\
\text { tipos de incidentes relatados foram } \\
\text { documentação, medicamentos, admi- } \\
\text { nistração clínica e processo clinico. }\end{array}$ \\
\hline 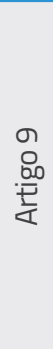 & $\begin{array}{l}\text { BMJ Quality \& Safety, } \\
2008\end{array}$ & $\begin{array}{l}\text { Kuo GM, Phillips RL, } \\
\text { Graham D, Hickner } \\
\text { JM }\end{array}$ & $\begin{array}{l}\text { Medication errors repor- } \\
\text { ted by US family phy- } \\
\text { sicians and their office } \\
\text { staff }\end{array}$ & Quantitativo & $\begin{array}{l}\text { Os principais erros identificados es- } \\
\text { tavam relacionados a medicação, } \\
\text { prescrição, administração, documen- } \\
\text { tação, dispensação e monitoramen- } \\
\text { to. } 72,4 \% \text { dos erros não atingiu os } \\
\text { pacientes e nenhuma morte foi rela- } \\
\text { tada. O profissional que mais impediu } \\
\text { que os erros chegassem aos pacien- } \\
\text { tes foi o farmacêutico. }\end{array}$ \\
\hline $\begin{array}{l}0 \\
\frac{0}{0} \\
\frac{00}{20} \\
\frac{20}{2}\end{array}$ & $\begin{array}{l}\text { Revista da Escola de } \\
\text { Enfermagem da USP, } \\
2014\end{array}$ & $\begin{array}{l}\text { Padoveze MC, Fi- } \\
\text { gueiredo RM }\end{array}$ & $\begin{array}{l}\text { O papel da atenção pri- } \\
\text { mária na prevenção de } \\
\text { infecções relacionadas à } \\
\text { assistência à saúde }\end{array}$ & Estudo teórico & $\begin{array}{l}\text { São indicados sete componentes es- } \\
\text { senciais para desenvolvimento de um } \\
\text { programa de prevenção de IRAS na } \\
\text { atenção primária: precauções padrão, } \\
\text { precauçções específicas, cuidado com } \\
\text { medicamentos e imunobiológicos, } \\
\text { saúde ocupacional, educação perma- } \\
\text { nente, auditorias e resposta rápida. }\end{array}$ \\
\hline 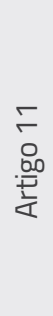 & $\begin{array}{l}\text { Revista Latino-Ameri- } \\
\text { cana de Enfermagem, } \\
2016\end{array}$ & $\begin{array}{l}\text { Paranaguá, TTB, } \\
\text { Bezerra ALQ, Tobias } \\
\text { GC, Ciosak SI }\end{array}$ & $\begin{array}{l}\text { Suporte para aprendiza- } \\
\text { gem na perspectiva da } \\
\text { segurança do paciente } \\
\text { na atenção primária em } \\
\text { saúde }\end{array}$ & Transversal & $\begin{array}{l}\text { Foram evidenciados pontos favoráveis } \\
\text { de suporte à aprendizagem, como res- } \\
\text { peito mútuo, autonomia para organizar } \\
\text { o trabalho e a valorização de novas } \\
\text { ideias. As variáveis que dificultam o } \\
\text { processo de aprendizagem no ambien- } \\
\text { te de trabalho foram a resistência às } \\
\text { mudanças e o excesso de serviço. }\end{array}$ \\
\hline
\end{tabular}




\begin{tabular}{|c|c|c|c|c|c|}
\hline 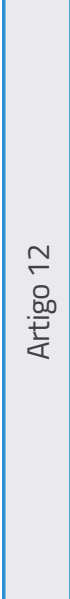 & $\begin{array}{l}\text { Cogitare Enfermagem, } \\
2016\end{array}$ & $\begin{array}{l}\text { Mesquita KO, Silva } \\
\text { LCC, Lira RCM, Frei- } \\
\text { tas CASL, Lira GV }\end{array}$ & $\begin{array}{l}\text { Segurança do paciente } \\
\text { na atenção primária à } \\
\text { saúde: revisão integra- } \\
\text { tiva }\end{array}$ & $\begin{array}{l}\text { Revisão inte- } \\
\text { grativa }\end{array}$ & $\begin{array}{l}\text { Observou-se que as publicações data- } \\
\text { vam de } 2008 \text { em diante, com temática } \\
\text { variando entre investigação da cultura } \\
\text { de segurança, relação da segurança do } \\
\text { paciente com a prevenção de eventos } \\
\text { adversos e avaliação de incidentes, e } \\
\text { tradução de instrumento para avalia- } \\
\text { ção da segurança do paciente. O conte- } \\
\text { údo evidenciado pela análise permitiu } \\
\text { concluir sobre a importância de discutir } \\
\text { sobre segurança de modo a contribuir } \\
\text { na melhoria da qualidade da assistên- } \\
\text { cia à saúde, assim como a necessidade } \\
\text { de novos estudos, haja vista relevância } \\
\text { do tema e pouca produção científica na } \\
\text { área }\end{array}$ \\
\hline
\end{tabular}

porcionar ao indivíduo o mais completo bem-estar. ${ }^{14}$ Nessa perspectiva, a APS deve ser considerada um componente-chave na área da segurança do paciente, visto que é responsável por coordenar uma resposta integrada a todos os níveis de atenção do sistema de saúde. ${ }^{15}$

Contudo, na atualidade, as pesquisas relacionadas à segurança do paciente estão predominantemente direcionadas para a assistência hospitalar; no entanto, a maior parte dos cuidados ocorre fora desse ambiente devido ao investimento do Brasil na implantação da Estratégia Saúde da Família (ESF) para reorganização e fortalecimento da APS. ${ }^{16-18}$

A Organização Mundial da Saúde (OMS), no ano de 2012, formou um grupo para estudar as questões relacionadas com a segurança do paciente na APS de modo que se avance no conhecimento sobre os riscos para os pacientes em cuidados de saúde primários e se aponte a magnitude e a natureza dos eventos adversos devido a práticas inseguras. ${ }^{19}$

Esse grupo, mediante revisão sistemática identificou que ainda não existe um método mais indicado para investigar a manifestação de eventos adversos na APS em países em desenvolvimento. ${ }^{20}$ Nos países desenvolvidos, os métodos mais utilizados para verificar eventos adversos na APS foram: avaliações dos eventos em sistemas de notificação, questionários, entrevistas e grupos focais. ${ }^{19-20}$

\section{Esse grupo,}

\section{mediante revisão}

\section{sistemática}

\section{identificou que}

ainda não existe

um método

mais indicado

para investigar a

manifestação de

eventos adversos na
APS em países em
desenvolvimento.

Estima-se que na APS a ocorrência de eventos adversos varia de 0,004 a 240 por mil consultas e os erros evitáveis variam de 45 a $76 \% .^{20}$ Em estudo brasileiro realizado em uma microrregião de saúde no estado do Rio de Janeiro identificou uma razão de eventos adversos de $1,1 \%$ em um total de 11.233 consultas na APS. Destes, $0,11 \%$ dos eventos adversos não chegaram a atingir os pacientes; $0,09 \%$ atingiram os pacientes, mas não causaram dano; e $0,91 \%$ atingiram os pacientes e causaram dano. ${ }^{10}$

O maior número de eventos adversos foi identificado em pacientes mais velhos - acima de 40 anos (83\%) e com doenças crônicas (68\%). Baseado nisso, em um estudo realizado em hospitais brasileiros identificou-se que a faixa etária acima de 60 anos tende a ser a que mais sofre com eventos adversos devido, justamente, ao aumento da prevalência de doenças crônicas e com a associação de comorbidades. ${ }^{21}$

Wallis e Dovey ${ }^{22}$ identificaram em seu estudo que os tipos de eventos adversos na APS foram relacionados principalmente devido ao atraso diagnóstico (16\%), medicação (38\%), tratamento odontológico (16\%), injeções e vacinas (10\%); Mira et al. ${ }^{23}$ encontraram como fator contribuinte mais frequente os erros relacionados à comunicação entre médico e pacientes (17,3\%); O’Beirne et al. ${ }^{24}$ identificaram eventos adversos relacionados à documentação $(41,4 \%)$ e processos administrativos (29,3\%); já Kuo et al. ${ }^{25}$ identificaram erros 
relacionados à medicação, sendo $70 \%$ relacionados a prescrição, $10 \%$ na administração, $10 \%$ no registro e $7 \%$ na dispensação.

$\mathrm{Na}$ revisão sistemática realizada por Marchon e Mendes Júnior ${ }^{19}$, os fatores contribuintes para os eventos adversos no âmbito da APS foram: falhas na comunicação interprofissional e com o paciente, falhas na gestão, tais como: falta de insumos médico-cirúrgicos e de medicamentos, profissionais pressionados para serem mais produtivos em menos tempo, falhas em prontuários e na recepção dos pacientes, planta física da unidade de saúde inadequada, descarte inadequado de resíduos da unidade de saúde, tarefas excessivas e falhas no cuidado. As falhas no cuidado envolveram falhas no tratamento medicamentoso (principalmente erro na prescrição), falha no diagnóstico, demora na realização do diagnóstico, demora na obtenção de informações e interpretação dos achados laboratoriais, falhas em reconhecer a urgência da doença ou suas complicações e déficit de conhecimento profissional.

Marchon, Mendes Júnior e Pavão ${ }^{10}$ identificaram que os fatores que contribuíram com os eventos adversos na APS foram $34 \%$ relacionadas a falhas no cuidado; $13 \%$ a falhas na gestão; e $53 \%$ a falhas na comunicação, sendo que $24 \%$ estavam relacionadas a falhas na comunicação com os pacientes, $19 \%$ na rede de atenção e $19 \%$ na comunicação interprofissional.

Os riscos associados aos procedimentos mais comuns realizados na APS são pouco conhecidos, com exceção daqueles relacionados à vacinação. Comumente são realizados procedimentos com certo grau de invasividade, como coleta de exames colpocitológico, inserção de dispositivo intrauterino (DIU), cauterização de colo uterino, glicemia capilar, inalações, curativos, administração de injetáveis, para os quais se pode antecipar algum evento adverso associado. Entretanto, até o momento não existem estimativas a este respeito. ${ }^{26}$

\section{Os riscos associados} aos procedimentos

\section{mais comuns}

realizados na

\section{APS são pouco conhecidos, com exceção daqueles relacionados à vacinação.}

Diante dos resultados apresentados e considerando que a APS é caracterizada como a porta de entrada do sistema de saúde e que tem a premissa de resolver cerca de $85 \%$ dos problemas da população, seus profissionais devem ter uma atuação assertiva de modo a prevenir a ocorrência de eventos adversos. ${ }^{27}$

Ressalta-se que para a prevenção de riscos é necessário identificar e analisar a sua origem, bem como sistematizar as medidas preventivas de forma proativa e, não somente, quando os erros acontecem. Estabelecer uma sistemática de gerenciamento de riscos é um caminho para a busca de melhor controle e monitoramento dos processos de trabalho. ${ }^{28}$

Reitera-se, assim, a necessidade de ampliar a cultura de segurança na APS de modo a possibilitar que pacientes e profissionais possam reconhecer e gerenciar os eventos adversos e serem sensíveis à sua capacidade compartilhada para a mudança reduzindo erros e tensões entre profissionais e a população. ${ }^{11}$

Portanto, cresce a importância do tema segurança do paciente na APS nas principais organizações internacionais de saúde. Nesse sentido, há espaço e há necessidade para realização de estudos sobre a temática.

\section{CONCLUSÃO}

Apesar da evolução na abordagem sobre a segurança do paciente, as investigações sobre eventos adversos na APS, ainda estão escassas no Brasil. Assim, a adoção de estratégias relacionadas à segurança do paciente precisa ser mais bem desenvolvida nesse nível de atenção, de forma a identificar quais as oportunidades e os desafios dos profissionais de saúde na prestação de uma atenção à saúde resolutiva, efetiva e de qualidade. A temática tem evoluído entre os profissionais de saúde, inclusive na alta liderança, o que estimula o envolvimento da instituição como um todo.

Portanto, os resultados deste estudo podem subsidiar discussões entre gestores e profissionais de saúde da APS a fim de identificar as necessidades e limitações para o fomento da segurança do paciente.

\section{REFERÊNCIAS}

1. Gama ZAS, Saturno-Hernandez PJ, Caldas ACSG, Freitas MR Mendonça AEO, Medeiros CAS et al. Questionário AGRASS: avaliação da gestão de riscos assistenciais em serviços de saúde. Rev Saúde Pública. 2020;54(21):1-15. doi: https://doi.org/10.11606/ s1518-8787.2020054001335
2. Raimondi DC, Bernal SCZ, Oliveira JLC, Matsuda LM. Patient safety culture in primary health care: analysis by professional categories. Rev Gaúcha Enferm. 2019;40(esp):e20180133. doi: https://doi.org/10.1590/1983-1447.2019.20180133

3. Silva EMA, Aguiar RS. Fatores relacionados à polimedicação 


\section{REFERÊNCIAS}

em idosos e a segurança do paciente: uma revisão integrative. Nursing (São Paulo). 2020;23(265):4127-4133. doi: https://doi. org/10.36489/nursing.2020v23i265p4127-413

4. Institute of Medicine. To err is human: building a safer health system. Washington (DC): National Academic Press; 1999.

5. Lemos GC, Azevedo C, Bernardes MFVG, Ribeiro HCTC, Menezes AC, Mata LRF. A cultura de segurança do paciente no âmbito da enfermagem: reflexão teórica. Rev. enferm. Cent.-Oeste Min. 2018; 8:e2600. doi: https://doi.org/10.19175/recom.v8i0.2600

6. Macedo LL, Silva AMR, Silva JFM, Haddad MCFL, Girotto E. The culture regarding the safety of the patient in primary health care: distinctions among professional categories. Trab Educ Saúde. 2020;18(1):e0023368. doi: https://doi.org/10.1590/19817746-sol00233

7. Lima CS, Aguiar, RS. Acesso dos homens aos serviços de atenção primária à saúde: uma revisão integrativa. Research, Society anda Development. 2020;9(4):e157943027. Doi: http:// dx.doi.org/10.33448/rsd-v9i4.3027

8. Marinho RAQC, Aguiar RS. A atenção primária como eixo estruturante da redução dos indicadores de violência contra crianças e adolescentes. REVISA. 2019;8(2):228-41. doi: https:// doi.org/10.36239/revisa.v8.n2.p228a241

9. Aguiar RS, Santana DC, Santana PC. A percepção do enfermeiro da estratégia saúde da família sobre a saúde do homem. Rev Enferm Cent O Min. 2015;5(3):1844-1854. doi: https://doi. org/10.19175/recom.v5i3.872

10. Marchon SF, Mendes Júnior WV, Pavão ALB. Caracteristics of adverse events in primary health care in Brazil. Cad Saúde Pública. 2015;31(11):2313-2330. doi: https://doi.org/10.1590/0102$311 \times 00194214$

11. Mesquita KO, Silva LCC, Lira RCM, Freitas CASL, Lira GV. Patient safety primary health care: an integrative review. Cogitare Enferm [Internet]. 2016 [cited 2020 Feb 19];21(2):0108. Available from: https://revistas.ufpr.br/cogitare/article/ view/45665/28526

12. Verbakel NJ, Van Melle M, Langelaan M, Verheij TJM, Wagner C, Zwart ADLM. Exploring patient safety culture in primary care. Int J Qual Heal Care [Internet]. 2014 [cited 2019 Out 10];26(6):585-91. Available from: https://www.ncbi.nlm.nih.gov/ pubmed/25085256

13. Oliveira JLC, Silva SV, Santos PE, Matsuda LM, Tonini NS Nicola AL. Segurança do paciente: conhecimento entre residentes multiprofissionais. Einstein (São Paulo) [Internet]. 2020 [citec 2020 Feb 19];15(1):50-7. Available from: https://www. scielo.br/pdf/eins/v15n1/pt_1679-4508-eins-15-01-0050.pdf

14. Donabedian A. An introduction to quality assurance in health care. New York: Oxford University Press; 2003.

15. Organização Mundial de Saúde. Relatório Mundial de Saúde 2008: cuidados de saúde primários agora mais que nunca. Lisboa: Ministério da Saúde; 2008.

16. Silva APF, Backes DS, Magnago TSBS, Colomé JS. Segu- rança do paciente na atenção primária: concepções de enfermeiras da estratégia de saúde da família. Rev. gaúch. enferm. 2019;40(esp):e20180164. doi: https://doi.org/10.1590/19831447.2019 .20180164

17. Souza LM, Silva MCS, Zavalhia SEM Coppola IS, Rocha BP. Percepção de enfermeiros da Estratégia de Saúde da Família sobre segurança do paciente. J. nurs. health, 2018;8(2):e188205 doi: https://doi.org/10.15210/jonah.v8i2.12721

18. Alves AS, Aguiar RS. Segurança do paciente no âmbito domiciliar: uma revisão integrativa. Research, Society and Development. 2020;9(3): e181932709. Avaiable from: https://rsd.unifei. edu.br/index.php/rsd/article/download/2700/2066

19. Marchon SG, Mendes Júnior WV. Segurança do paciente na atenção primária à saúde: revisão sistemática. Cad. Saúde Pública. 2014;30(9):1-21. doi: https://doi.org/10.1590/0102311X00114113

20. Makeham M, Dovey S, Runciman W, Larizgoitia I. Methods and measures used in Primary Care Patient safety research. Dunedin, NZ: World Health Organization; 2008.

21. Mendes Júnior WV, Pavão ALB, Martins M, Moura MLO, Travassos C. The feature of preventable adverse events in hospitals in the State of Rio de Janeiro. Rev. Assoc. Med. Bras. 2013;59(5):421-8. doi: http://dx.doi.org/10.1016/j. ramb.2013.03.002

22. Wallis K, Dovey S. No-fault compensation for treatment injury in New Zealand: identifying threats to patient safety in primary care. BMJ Qual Saf. 2011;20:587-91. Available from: https:// pubmed.ncbi.nlm.nih.gov/21228439/

23. Mira JJ, Nebot C, Lorenzo S, Pérez-Jover V. Patient report on information given, consultation time and safety in primary care. BMJ Qual Saf. 2010;19(5):e33. Available from: https://pubmed. ncbi.nlm.nih.gov/20511244/

24. O'Beirne M, Sterling PD, Zwicker K, Hebert P, Norton P. Safety incidents in family medicine. BMJ Qual Saf. 2011;20(12):100510. Available from: https://pubmed.ncbi.nIm.nih.gov/21893612/

25. Kuo GM, Phillips RL, Graham D, Hickner JM. Medication errors reported by US family physicians and their office staff. BMJ Qual Saf. 2008;17(4):286-90. Available from: https://qualitysafety. bmj.com/content/17/4/286

26. Padoveze MC, Figueiredo RM. O papel da Atenção Primária na prevenção de Infecções Relacionadas à Assistência à Saúde. Rev. Esc. Enferm. USP. 2014;48(6):1137-44. doi: https://doi. org/10.1590/S0080-623420140000700023

27. Paranaguá TTB, Bezerra ALQ, Tobias GC, Ciosak SI. Suporte para aprendizagem na perspectiva da segurança do paciente na Atenção Primária à Saúde. Rev. latino-am. enferm. 2016;24(e2771):01-09. doi: http://dx.doi.org/10.1590/1518-8345.0784.2771

28. Hinrichsen SL, Oliveira CLF, Campos M, Possas L, Sabino G, Vilella TAS. Gestão da Qualidade e dos riscos na segurança do paciente: estudo-piloto. RAHIS. 2011;7(7):10-7. doi: https://doi. org/10.21450/rahis.v3i7.1400 